\title{
Simultaneous Body Part and Motion Identification for Human-following Robots
}

\author{
Sihao Ding ${ }^{\mathrm{a}}$, Qiang Zhai ${ }^{\mathrm{b}}$, Ying $\mathrm{Li}^{\mathrm{a}}$, Junda Zhu'c ${ }^{\mathrm{c}}$, Yuan F. Zheng ${ }^{\mathrm{a}}$, and Dong Xuan ${ }^{\mathrm{b}}$ \\ ${ }^{a}$ Dept. of Electrical and Computer Engineering, The Ohio State University, USA. \\ \{dings, liyi, zheng\}@ece.osu.edu \\ ${ }^{b}$ Dept. of Computer Science and Engineering, The Ohio State University, USA \\ \{zhaiq,xuan\}@cse.ohio-state.edu \\ ${ }^{c}$ Dept. of Electrical and Computer Engineering, University of Macau, China. \\ jdzhu@umac.mo
}

\begin{abstract}
Human-following robots are important for home, industrial and battlefield applications. To effectively interact with human, a robot needs to locate a person's position and understand his/her motion. Vision based techniques are widely used. However, due to the close distance between human and robot, and the limitation in a camera's field of view, only part of a human body can be observed most of the time. As such, the human motion observed by a robot is inherently ambiguous. Simultaneously identifying the body part being observed and the motion the person undergoing is a challenging problem, and has not been well studied in the past. In this paper, we propose a novel method solving the body part and motion identification problem in a unified framework. The relative position of an observed part with respect to the whole body and the motion type are treated as continuous and discrete labels, respectively, and the most probable labeling is inferred by structured learning. A fast part-distribution estimation is introduced to reduce the computational cost. The proposed approach is able to identify different body parts without explicitly building models for each single part, and to recognize the motion with only partial body observations. The proposed approach is evaluated using actual videos captured by a human-following robot as well as the synthesized videos from the public UCF50 dataset, originally developed for action recognition. The result demonstrates the effectiveness of the approach. Keywords: partial body identification, motion recognition, structured learning
\end{abstract}




\section{INTRODUCTION}

With recent active research, human-following robots $[1,2,3,4,5]$ that assist and cooperate with people have been introduced to different applications across a wide range of fields. There are robot followers for senior care, disability assist, telepresence, and even following soldiers in battlefields [6]. With cameras equipped, the robots are able to capture videos and perform vision-based human-robot interactions (HRI).

Computer vision can play a huge role in such applications. To understand the motion being performed by humans is of fundamental importance to HRI. Due to the egocentric view and close distance, however, a human-following robot can often observe only part of the human body. As some examples of the egocentric view shown in Figure 1, the limited perception of the human body can cause difficulty in many vision applications such as motion recognition. It is desirable that a human-following robot is able to recognize the motion being performed even with limited perception of the human body. On the other hand, correctly identifying the body parts being observed can be useful in many HRI tasks such as determining the relative position between human and robot, human pose recognition, and specific body part tracking.

In this paper, we address the problem of simultaneously identifying partially observed human body as well as the human motion. In addition to the difficulty of motion recognition, there are several facts that make this problem even more challenging.

The first is the completeness and continuity of the part being observed. It is unlikely for the robot to always observe discrete and well-segmented parts, like "head" or "legs". In practice, parts like "head and left shoulder" or "part of legs below knees" will show up in the view of camera, which makes it difficult to build separate models for well defined body parts. The second challenge is on part-motion ambiguity. Since the person being followed may present different motions such as walking and running, a same body part with different motions can look different while different body parts with different motions may be similar. For such ambiguity, the identification of part and motion should be considered simultaneously, but not independently. Finally, this task is very different from distinguishing different body parts from a fully observed whole body. Only a partial body is observable by the robot during the part identification, which means 


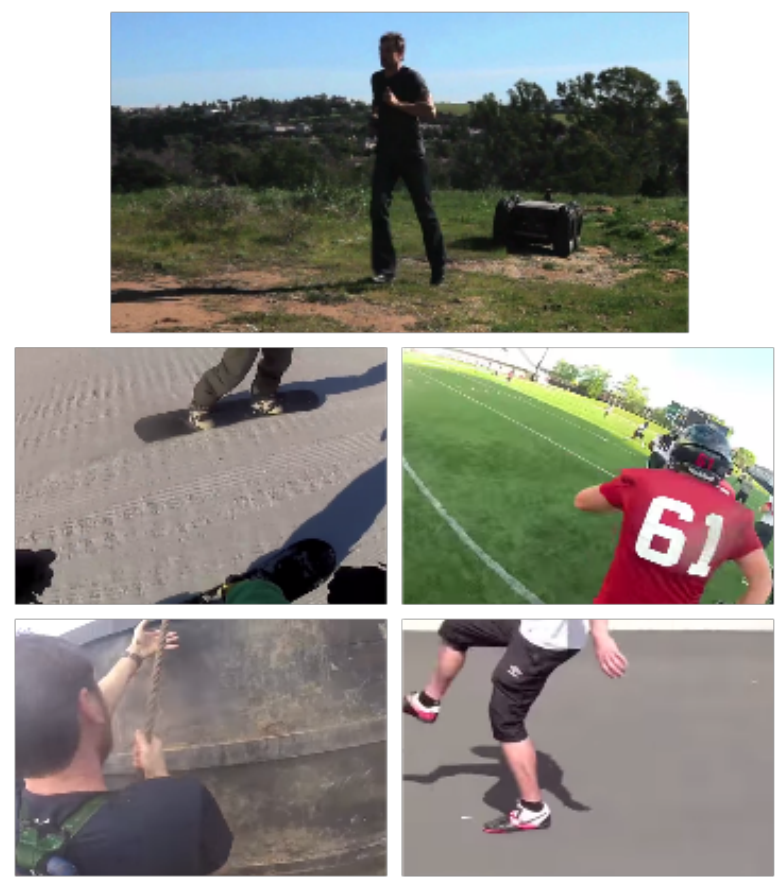

Figure 1: Examples of human-following robot (top row) and partially observed human body with motion in egocentric view (second and third row).

that it is not possible to use of the unobserved whole body as a valuable reference. As opposed to locate predefined body parts in a given full body, we are given an observed part and trying to identify which part it is. Due to the facts stated above, the body part being identified lies in a continuous space instead of a limited collection. Specifically, we want to identify the relative position of an observed part with respect to the whole body.

Throughout this paper, "motion" refers to the movements of human which last for or can be identified within a short period of time, such as jumping and walking; complex activities with longer duration such as playing basketball games and weddings are not considered. We also assume that the human-following robots observe human beings from behind which produces egocentric videos with back view or semi-back view of human bodies. The motion categories considered here are limited and discrete.

To counter the challenges, we propose using a structured learning framework to simultaneously infer the observed body part and the motion. The overview of our approach is shown 
in Figure 2. The training videos are evaluated against spatial-temporal volumes sampled from reference videos specified by the labels of the training samples. A structured support vector machine (SVM) is trained using the evaluation results, guided by a fast part-distribution estimation. The labels of the testing videos are determined using the trained model as well as the reference videos. The approach is able to identify different body parts without explicitly building separate models for each single part. Furthermore, it can identify the whole body motion from the observed body parts.

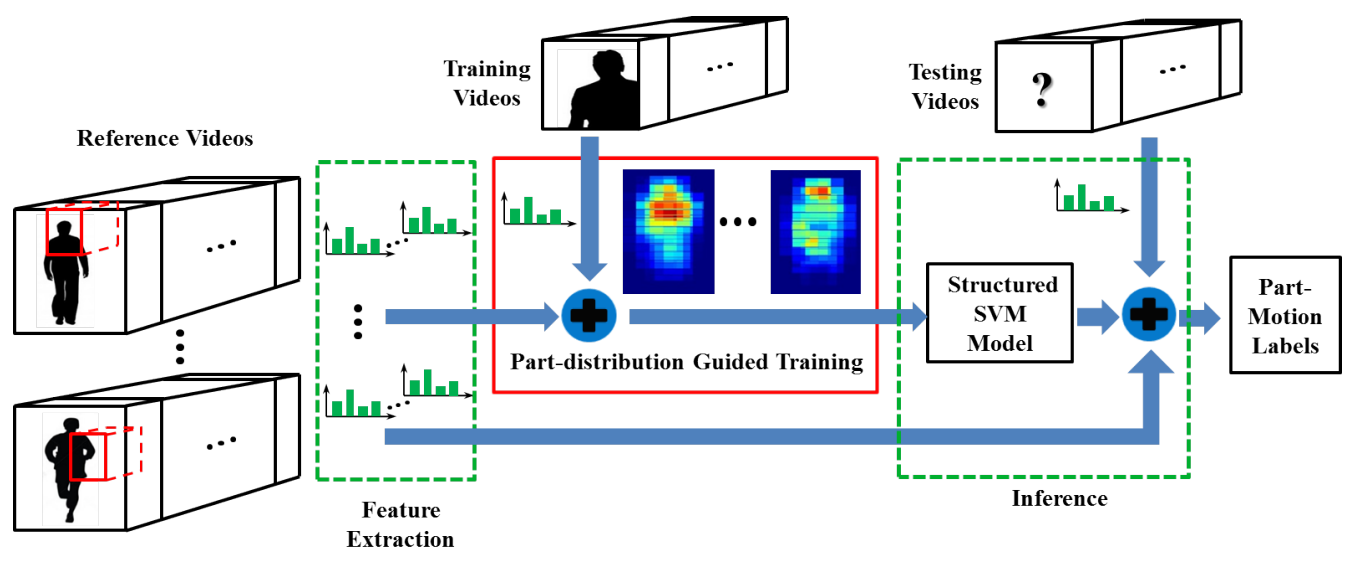

Figure 2: Overview of the proposed approach.

The rest of the paper is organized as follows: we examine the relevant literature in Section 2, our design rationale is discussed in Section 3, the details of the proposed approach are described in Section 4, the approach is evaluated and experiment results are presented in Section 5, and the paper is concluded in Section 6.

\section{RELATED WORK}

\subsection{Human-following Robots}

Human-following robots have been studied for long, and various methods have been proposed to achieve successful following. Cosgun et al. [1] combined human tracking and motion planning to enable autonomous person following for telepresence robots. Horiuchi et al. [2] used a laser range finder to track pedestrian while Kobilarov et al. [3] used laser together with an 
omni-directional camera to do the job. Gockley et al. [5] designed robots that behave socially appropriately while following human beings.

For human tracking purpose, Satake et al. [4] built depth templates for head, and detect and follow human beings using stereo vision on the robot. In [7], Brookshire et al. proposed to follow persons using histograms of oriented gradients, in which the whole body model is trained and the human is detected using the model. However, these approaches are limited to situations where the head or the whole human body must be observable in order to apply the specific models.

\subsection{Specific Part Model-based Detection and Part Identification from Full Body}

There are works that detect specific human body parts based on part-specific models. Ardiyanto et al. proposed in [8] an upper body orientation estimation method, which is based on partial least square. An upper body model is trained with the histograms of oriented gradients (HOG) and the local binary pattern (LBP) features. Wu et al. [9] used a combination of multiple specific part detectors to detect partially occluded human bodies. Not being designed specifically for part identification tasks, these methods require separate training for different body parts and can face difficulty when dealing with randomly appeared, not trained body parts. Based on static images, the methods do not consider the impact of different motions imposed on the appearance of the body parts.

Srinivasan et al. introduced a bottom-up method for recognition and parsing of the human body in [10]. Plagemann et al. [11] proposed a body part identification and localization method using depth images. Similar to the deformable part model (DPM) [12], they utilized the whole body information when detecting different parts. The application scenarios of these methods, in which full human bodies are observed and different parts are identified from them, are different from ours. Eichner et al. [13] proposed a method for estimating body part appearance models for pictorial structures, which improved existing articulated human pose estimation algorithm. Sapp et al. [14] presented a multimodal approach that captured different pose modes for pose estimation. In the follow up work by Jain et al. [15], motion information was integrated into the appearance information, based on a deep learning framework. The experiments showed that the introduction of motion features greatly improved the performance. The focus of these works 
is pose estimation, more specifically, "where" a particular body part is, given data that contains that part. Differently, our focus is to determine "what" the body part is, given only the partially observed body.

\subsection{Action/Motion Recognition}

The study on action/motion recognition has been paid significant attention in the past decade. Some works focused on the construction of spatio-temporal features like space-time interest points (STIP) [16], Cuboid [17] and dense trajectories [18], to name a few. Recently, Zhu et al. [19] integrated context information into activity recognition. Oneata et al. proposed in [20] to encode appearance and motion feature using Fisher Vector. Pirsiavash et al. [21] parsed videos by localizing the temporal segments using segmental grammars. Gong et al. [22] collected a still image dataset which are dedicated to interactions between people. A more related work to ours by Cao et al. [23] looked into action recognition from partially observed videos. Different from our work, the concept of "partially observed" is in temporal instead of spatial domain. It attempted to recognize action from temporal truncated videos, while our work focuses on predicting whole body motion from partially observed body.

\subsection{Egocentric Vision}

Egocentric (first person vision) videos produced by sources such as robot-mounted cameras and wearable cameras have attracted a large amount of attention recently. A survey can be found in [24]. Focus has been on object recognition (e.g. [25, 26]), action recognition (e.g. [27, 28]), and video summarization (e.g. [29, 30]).

Some works look into specific body parts, especially hands of camera wearer's, in egocentric videos. Betancourt et al. [31] proposed a sequential classifier to improve true negatives in hand detection. In [32], Lee et al. studied how to distinguish different pairs of hands of the camera wearer and others. Hands detection usually includes skin color modeling, which is not always applicable to the other body parts. 


\subsection{Structured SVM Framework and Applications}

Structured SVM is proposed by Tsochantaridis et al. [33]. Its ability to predict structured output has gained itself large potential to solve complex problems. Blaschko et al. [34] formulate object detection problem as a structured output regression. Hu et al. [35] applied structured SVM to recognition of soft-labeled activities, which are regard as a sequential labeling problem. Bertelli et al. [36] proposed a supervised object segmentation method using kernelized structured SVM.

\section{DESIGN RATIONALE}

In this section we introduce the design rationale of the algorithm, in particular, structured learning and why the problem can be formulated in such a way.

\subsection{Structured Learning}

Structured learning is to learn to predict outputs that are structured in the output space from input data. Given $n$ pairs of training data and corresponding labels $\left\{x_{i}, y_{i}\right\}$ for $i=$ $1, \ldots, n, x_{i} \in \mathcal{X}, y_{i} \in \mathcal{Y}$, a mapping of $\mathcal{X} \longmapsto \mathcal{Y}$ is learned and is applied to new data to predict their labels.

Structured SVM learns the mapping from training samples by solving the following optimization problem with a set of linear inequality constraints:

$$
\begin{array}{ll}
\min _{w, \xi} & \frac{1}{2}\|w\|^{2}+C \sum_{i=1}^{n} \xi_{i} \\
\text { s.t. } \quad & \xi_{i} \geq 0, \forall i \\
& \left\langle w, \Psi\left(x_{i}, y_{i}\right)\right\rangle-\left\langle w, \Psi\left(x_{i}, y\right)\right\rangle \geq \Delta\left(y, y_{i}\right)-\xi_{i}, \\
& \forall i, \forall y \in \mathcal{Y} \backslash y_{i},
\end{array}
$$

in which $w$ denotes a parameter vector, $\xi_{i}$ is the slack variable for the $i^{t h}$ sample and $C$ is the soft margin parameter. $\Psi\left(x_{i}, y_{i}\right)$ is a feature function describing some joint characteristics of a 
given data and its label, which is application dependent. The loss function $\Delta\left(y, y_{i}\right)$ measures a distance between $y$ and $y_{i}$ in the label space which satisfies $\Delta\left(y, y_{i}\right) \geq 0$ and $\Delta(y, y)=0$.

The inference of a label $y \in \mathcal{Y}$ from a given data $x \in \mathcal{X}$ and the trained $w$ follows the following equation:

$$
f(x)=\underset{y}{\arg \max }\langle w, \Psi(x, y)\rangle .
$$

\subsection{Problem Setting}

In our defined problem, the output space is the combination of the part of the human body observed and the motion categories. In the form of the relative position with respect to the whole body, the observed body part lies in a continuous space which we wish to describe quantitatively, while the motion category can be represented by discrete labels. These two variables together define a structured output. To represent the body parts quantitatively, a global canonical coordinates is set up to describe their positions and area in the form of bounding boxes. All the training labels should be generated from or aligned to the canonical coordinates so that the same bounding box will indicate the same partial body. For the data, any reasonably designed features extracted from the videos will and should be affected by the part of the body appearing in the video as well as the motion being performed. Such features and their corresponding body part and motion can compose the pairs of data and labels in a structured learning framework.

\subsection{The Reference Set}

We introduce a reference set into our data during both training and testing processes, to "reference to" and "evaluate against", for training and testing videos. To understand why this is necessary, we first elaborate the difference between the object detection problem and the current problem of part-motion identification.

Image-based object detection is seemingly similar to our current problem, yet inherently different. Given the training samples, a usual formulation [34] of the structured SVM is that the data $(x)$ is the feature extracted from training images and the label space $\mathcal{Y}$ is the bounding boxes at all possible locations and scale. The feature function $\Psi\left(x_{i}, y_{i}\right)$ can be some extracted features from the image $x_{i}$ within the bounding box specified by label $y_{i}$. During the testing process, a 
maximizer is found for (4) across all the possible bounding boxes $y$, to make the decision. For object detection, the problem is given a target (object to be detected) and a whole searching set (image), trying to identify a best match among its subsets (bounding boxes). On the other hand, our defined problem is given an observed subset (partially observed moving body), to identify which subset it is among the whole set (which part of the body) and indicate the state of the whole set (motion state).

If the same formulation as object detection is followed, it will end up with (4) for a similar searching space $\mathcal{Y}$. For object detection problem it is straight forward to searching through the testing image for the result. In our problem, however, we have no access to the whole set which the observed subset comes from, to search for. To address this issue, a reference set is introduced. Instead of using the extracted features from the subset directly as data $(x)$, we evaluate them against the particular reference set that are specified by their labels, and make the result of evaluation as the data. Accordingly, during the testing process, the label space $\mathcal{Y}$ now refers to the subsets of the reference set. This allows us to solve the inference problem in (4) with a feasible support of $\mathcal{Y}$. We will describe the details of the algorithm in the following sections.

\section{TECHNICAL APPROACH}

To facilitate the description of the approach, we first define the notations and setups which we will use in the following discussion. The testing video stream containing partially observed bodies will be packed into short video slices $\left\{V_{I}\right\}$ with fixed length $l$ for every $\Delta l$ frame. $\Delta l$ is set to be strictly smaller than $l$ so that the video slices will have overlap with neighboring ones. Though a person may show different parts in the view across the video, the part being observed within a video slice is treated as unchanged. So the part and motion identification is performed in a slice-wise manner. A database with multiple full body videos for each of the $M$ different motions is used for training. They are further diced into video cubes to generate data and label pairs. There is another set of full body observed videos used as reference videos. We denote the category of an motion $i$ as $m_{i}(i=1, \ldots, M)$. The training videos for one particular motion $m_{i}$ are denoted as $\left\{V_{m_{i}}\right\}$, and the reference videos with motion $m_{i}$ are denoted as $\left\{R_{m_{i}}\right\}$. Different 
body parts $p_{i}$ are represented by their pixel coordinates of the bounding boxes in the aligned video frames.

\subsection{Multi-scale Dense Sampling and Alignment}

In this section, the Multi-scale Dense Sampling and the human body alignment are introduced. They are applied to full body videos to generate labeled training samples, and testing samples with ground truth labels which are used later in the testing phase. We will also use this sampling scheme when computing the fast part-distribution estimation. To perform multi-scale dense sampling on videos, first the image pyramids of each frame in all the videos are computed. We obtain a multilevel video pyramid with a scaling factor to deal with size variation of the body parts appearing in the testing videos. Next, dense sampling in both spatial and temporal domains is applied to each scale level of the video pyramid. Each sample is a 3D spatial-temporal video volume with spatial size $w \times h$ and temporal length $l$. The spatial sampling stride is set to be $\Delta w<w$ and $\Delta h<h$, while the temporal sampling strides following the testing input video to be $\Delta l<l$. All the sampled video volumes have overlaps with their neighbors in both spatial and temporal domains. Each sampled video volume contains a small portion of the appearance and motion information of the holistic video.

Since both the robot and the person being followed are moving, it is hard to keep a constant relative position between the two. Thus the human body may appear in different image locations both between and within the training videos, which is undesirable. In order to represent body parts by their pixel coordinates, all the training videos (thus their dense sampled cubes) and reference videos must be aligned so that the same body part locates in the same position of the frame across all the frames of the videos.

One "anchor" frame is pulled out at every $\Delta l$ frames from the training videos. Human body in each "anchor" frame is marked by a rectangular bounding box. A canonical rectangle is defined and all the bounding boxes are aligned to it. The pixel coordinates $(u, v)$ within the bounding boxes of the "anchor" frames are transformed into the aligned coordinates $p=(\hat{u}, \hat{v})$ by the 
following equations:

$$
\left\{\begin{array}{l}
\hat{u}=\left(u-u_{t l}\right) S_{w}+u_{t l}+u_{\text {shift }} \\
\hat{v}=\left(v-v_{t l}\right) S_{h}+v_{t l}+v_{\text {shift }}
\end{array}\right.
$$

in which $u_{t l}, v_{t l}$ are the coordinates of the top left pixel locations of the human body bounding box; $u_{\text {shift }}, v_{\text {shift }}$ are the translations of the left top corners between the human body bounding box and the canonical bounding box in the width and height directions, respectively; and $S_{w}$ and $S_{h}$ are the scales between them for width and height. The width and height of the original bounding box will be aligned to $\hat{w}=w \cdot S_{w}$ and $\hat{h}=h \cdot S_{h}$.

We assume that the human body can be viewed as static or with little movement in a very short time period. So the $(\Delta l-1)$ frames after each "anchor" frame share the same alignment as their corresponding "anchor" frame. To avoid breaking the continuity of the video, the alignment is performed only after the low-level feature of the samples are extracted. Only the spatial-area that are marked as human bounding boxes are sampled during the dense sampling process, for reducing computational burden and increasing accuracy. Unless specified otherwise, we assume that the alignment is performed to all the data in the training process.

\subsection{Formulation}

\subsubsection{Data (x) and Label (y)}

With previous densely sampled spatial-temporal cubes, we can now compose data-label pairs for the structured learning purpose. Instead of using the raw video cubes, we extract feature vectors from them as data. To counter the part-motion ambiguity, the feature should reflect the impact of both the appearance of the body part and the motion status. We apply dense trajectories (DT) coupled with Histograms of Oriented Gradients (HOG) and Motion Boundary Histograms (MBH) $[18,37]$ as the feature representation for the testing video as well as the sampled volumes. The trajectories of the interest points obtained from the dense optical flow field are tracked through the video, and the $\mathrm{HOG}$ and $\mathrm{MBH}$ around these dense trajectories together with the trajectories themselves are used to describe the video. While this feature representation initially serves as an effective motion recognition method, the HOG and MBH components 
also have the ability to capture the edge information and moving boundaries of the objects, respectively. Though MBH is known to be insensitive to camera motion to some extent [38], recent study shows that it can still benefit from global camera motion compensation [37], which also helps produce better trajectories. The camera motion is modeled as a homography [39] between two successive frames. The homography is estimated using random sample consensus (RANSAC) [40] and the optical flow between two consecutive frames is computed after the second frame is transformed by the estimated homography.

The low-level features are encoded using a standard Bag-Of-Words (BOW) [41] method. Features extracted from the training videos are used to train the visual vocabularies with $k$-means clustering. A histogram of $n$ visual word occurrences is computed for each video. This histogram is used as the data vector in our structured SVM framework.

For a datum $x$, the corresponding label $y$ as a combination of part $(p)$ and motion $\left(m_{i}\right)$ is defined as a four-dimensional vector:

$$
\begin{aligned}
y & =\left[p, m_{i}\right] \\
& =\left[u_{t l}, v_{t l}, s, m_{i}\right]
\end{aligned}
$$

in which $u_{t l}$ and $v_{t l}$ is the pixel location of the top left corner of the bounding box, $s$ is the pyramid level, and $m_{i}$ indicates the class the motion belongs to. The bounding boxes are all with a fixed width-height ratio in our implementation. During the dense sampling process, all the aligned bounding boxes and motion categories are recorded with the associated spatial-temporal cubes, so that we know the correspondence between data and labels for training. For each pair $\left(x_{i}, y_{i}\right)$, we have:

$$
x_{i}=F\left[V\left(y_{i}\right)\right],
$$

in which $V\left(y_{i}\right)$ is a simplified notation of $V_{m_{i}}\left(p_{i}\right)$, and $F[\cdot]$ is the low-level feature extraction operator. This means that, a piece of data $x_{i}$ is a low-level feature representation of a video cube, which is located at $p_{i}$ in video $V_{m_{i}}$. Here, the mapping from data to label is unique, while it's not the true for the opposite case. Given a $x_{i}$, its label $y_{i}$ is uniquely defined by its position in the 
aligned coordinates as well as the motion category of the video. Since a label $y_{i}$ only specifies the motion category $m_{i}$ instead of the exact videos belong to that motion category, the feature representations of the same body part (aligned) in different videos with the same motion share the same label.

\subsubsection{Feature Function}

Feature function $\Psi(x, y)$ should describe joint characteristics of a data-label pair. In our case, it is chosen to reflect the similarity between feature vectors of a video and that of reference videos within bounding box specified by the label, using various chosen distance measurements.

In a broad way, the overall data used for training consist of two kinds of videos: the videos containing partially observed human body $\{V(y)\}$, and the reference videos showing fully observed human body $\{R\}$. The $\{V(y)\}$ are obtained by cropping spatial-temporal video-cubes from fully observed videos with known motion categories at known bounding boxes, i.e., for all $y$. For simplicity, such a spatial-temporal cube is denote as $V_{c}$ in the following context. The reference videos consist of aligned full body videos of each motion. The number of reference videos of any particular motion is the same. For a training datum $x_{i}$ extracted from a cube $V_{c}$, it is compared with the cubes sampled from all the reference videos that belongs to the motion category $m_{i}$ within the bounding box $\left[u_{t l}, v_{t l}, s\right]$, where the motion category and the bounding box are specified by associated label $y_{i}$. Following previously defined notations, the reference video of motion $m_{i}$ and sampled at $p$ is denoted as $R(y)$. Given the part and motion (i.e., label $\left.y_{i}\right)$, the similarity score $\varphi$ between $x_{i}$ and $R\left(y_{i}\right)$ is defined as:

$$
\begin{aligned}
\varphi_{j, k, n}\left(x_{i}, y_{i}\right) & =\operatorname{Sim}_{k}\left\{x_{i} \cdot f_{n}, F\left[R_{j}\left(y_{i}\right)\right] \cdot f_{n}\right\} \\
& =\operatorname{Sim}_{k}\left\{F\left[V_{c}\right] \cdot f_{n}, F\left[R_{j}\left(y_{i}\right)\right] . f_{n}\right\},
\end{aligned}
$$

in which $\operatorname{Sim}_{k}$ is the similarity measurements, described by different distance measurements or their reciprocal, including Euclidean distance, Chi-Squared $\left(\chi^{2}\right)$ distance and correlation in our implementation; $f_{n}$ is one of the low-level features extracted from the videos including DT,

HOG and MBH. $R_{j}$ is the $j^{t h}$ reference video, and these reference videos can be different video 
slices from one long video, or from different videos. Each of such similarity score $\varphi$ fills one dimension of the vector of the feature function $\Psi$. For one data $\left(x_{i}\right)$-label $\left(y_{i}\right)$ pair, the feature function $\Psi\left(x_{i}, y_{i}\right)$ is:

$$
\Psi\left(x_{i}, y_{i}\right)=\left[\varphi_{0,0,0}\left(x_{i}, y_{i}\right), \ldots, \varphi_{J, K, N}\left(x_{i}, y_{i}\right)\right]^{T}
$$

in which $J, K$ and $N$ are the maximum number of $j, k$ and $n$. Every $\Psi\left(x_{i}, y_{i}\right)$ as shown in (9) results in a column vector with a dimension of $J \cdot K \cdot N$.

When a video with body partially observed in unknown motion is to be tested, the lowlevel feature extraction is performed to obtain low-level feature representations $x$ of it. The $x$ consists of multiple kinds of low-level features (in our case: DT, HOG and MBH). Given a label $y=\left[p, m_{i}\right]$, to compute the feature function $\Psi(x, y)$, a group of $J$ reference videos $R$ which belong to motion category $m_{i}$ is selected. Then, the low-level features at position $p$ are extracted for every selected reference video. After that, the similarity between these low-level features of reference videos and $x$ is measured, using different distance measurements. When an $x$ is paired with different label $y$, different $\Psi(x, y)$ can be computed by going through all groups of reference videos categorized by $m_{i}$, all bounding boxes at different positions, and all the different kinds of low-level features and similarity distance measurements.

To summarize, the value of the feature function alters when combining data $x$ with different label $y$. This is achieved by evaluating data against reference data that are drawn from different motion categories at different bounding boxes, specified by label $y$.

\subsubsection{Loss Function}

Loss Function $\Delta\left(y_{1}, y_{2}\right)$ is a non-negative function and should characterize the degree of correctness for both body part and motion identification. To reflect the loss of incorrect position of body parts, the overlap rate of two bounding boxes is chosen to be the loss function for this part. For robustness, the function is bounded by 1 , meaning that the loss is considered same as long as no overlap with correct bounding box is observed, and it decreases monotonically as the 
overlap area increases. The loss function for the body part is described as:

$$
\Delta_{p}\left(y_{1}, y_{2}\right)=1-\frac{A\left(y_{1} \cap y_{2}\right)}{A\left(y_{1} \cup y_{2}\right)},
$$

where $A(\cdot)$ is the overlapping area.

The second part of the loss function is the loss of incorrect motion categorization. This is modeled as a zero-one loss function as shown below:

$$
\Delta_{m}\left(y_{1}, y_{2}\right)= \begin{cases}0 & \text { if } m_{1}=m_{2} \\ 1 & \text { if } m_{1} \neq m_{2}\end{cases}
$$

where $m_{1}$ and $m_{2}$ are motion categories in label $y_{1}$ and $y_{2}$, respectively.

The total loss function is the summation of the loss for body part and the loss of motion:

$$
\Delta\left(y_{1}, y_{2}\right)=\Delta_{p}\left(y_{1}, y_{2}\right)+\Delta_{m}\left(y_{1}, y_{2}\right)
$$

In fact, there are many choices in designing the combination of loss for part and loss for motion. The two terms can multiply different weight coefficients associated with them, to adjust whether to emphasis accuracy of part identification or motion identification. Alternatively, one can also use the product of the terms to avoid assigning or learning different weight coefficients. In our implementation, we use (12) which has equal weight for part and motion losses.

\subsubsection{Find the Most Violated Constraint}

During the optimization, the most violated constraint is to be found at each iteration to approach the optimize $w$. It is defined as:

$$
\underset{y}{\arg \max } \Delta\left(y, y_{i}\right)+\left\langle w, \Psi\left(x_{i}, y\right)\right\rangle .
$$

Though the size of the motion category is limited, the amount of possible bounding boxes is too huge to do exhaust searching. We propose to speed up the searching process by a fast part- 
distribution estimation. For each sampled training video volume, a coarse part-distribution which indicates their rough positions in the canonical human body is estimated.

\section{Similarity Map:}

A similarity map (SiMap) is defined as a matrix with the same size as a frame from the reference videos, whose elements represent the likelihood of the corresponding pixels being observed in a testing video. To begin with, the multi-scale sampling is performed on the reference videos, at a relatively large sampling stride. A modified version of similarity score $\varphi$ using in (8) is defined as:

$$
D_{j, k}\left(x_{i}, y_{i}\right)=\operatorname{Sim}_{k}\left\{F\left[V_{c}\right] . f, F\left[R_{j}\left(y_{i}\right)\right] . f\right\},
$$

in which the modification is that the different kinds of low-level features are concatenated as one $f$, instead of separate $f_{n}$, to measure the similarity. The modified similarity score $D$ is evaluated between the sampled training video volume and all the sampled reference video volumes. For a particular training video volume, one similarity score will be obtained at each of the sampled location of reference videos. These similarity scores will be assigned to all the pixels which the sampled reference video volumes occupy. The pixel positions will further be aligned using (5) described in Section 4.1. For different levels of the video pyramid, they are also back scaled to the base level (scale equals to 1 ). The similarity map of the motion category $m_{i}$, using the $k^{\text {th }}$ similarity measurement, is computed as described in Algorithm 1.

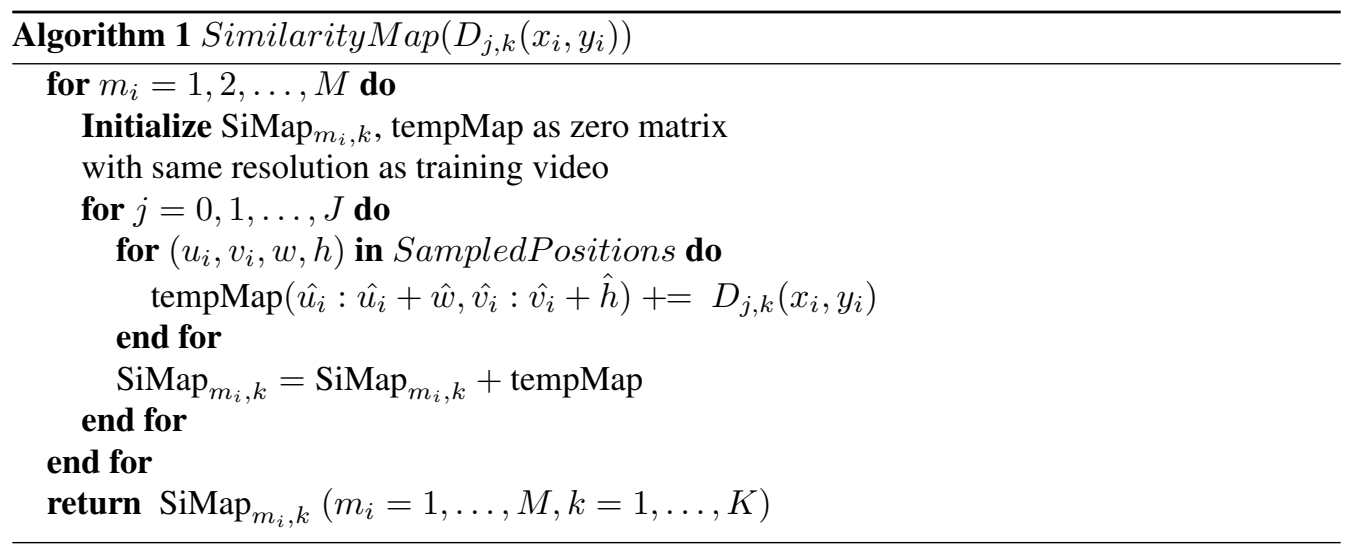

The similarity map reflects the overall cumulated similarity for all the parts $p$ represented by 
pixel locations. A visualization of the similarity map (with virtual human body overlaid) of the upper body in walking status evaluated against the walking reference videos is shown in Figure 3.

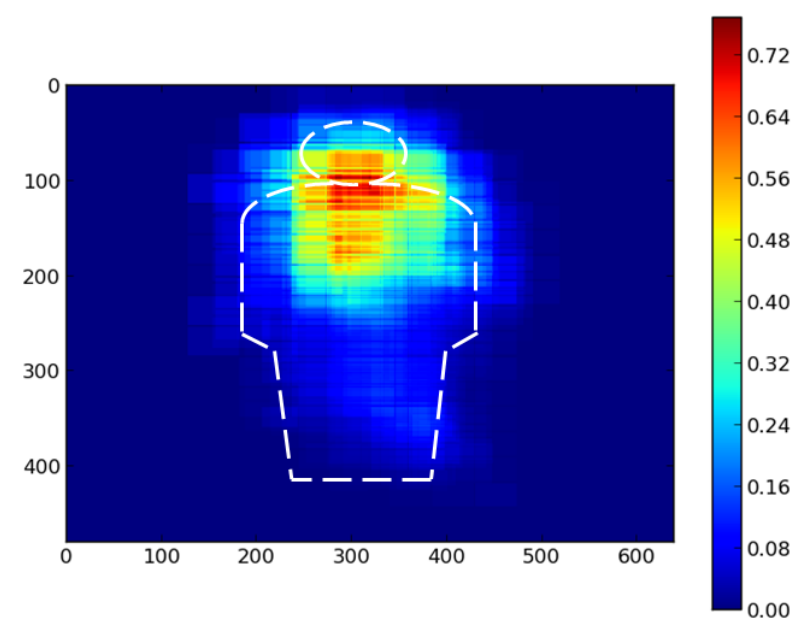

Figure 3: Visualization of the similarity map with virtual human body overlaid (similarities represented in colormap, best viewed in color).

\section{Part-distribution:}

With similarity maps for different motion categories, a joint distribution for part and motion can be obtained. When an input testing video is observed, the probability of the observed part being $p_{j}$ with motion status $m_{i}$ can be calculated as:

$$
P_{k}\left(m_{i}, p_{j}\right)=\frac{\operatorname{SiMap}_{m_{i}, k}\left(p_{j}\right)}{\sum_{i=0}^{M} \sum_{j=0}^{W \times H} \operatorname{SiMap}_{m_{i}, k}\left(p_{j}\right)},
$$

in which $W \times H$ is the resolution of the similarity map as well as the training video. Taking all the motion statuses into account, $P_{k}\left(m_{i}, p_{j}\right)$ depicts the probability of any pair of "part" and "motion" combined together. Again, each position $p_{j}$ in every motion status $m_{i}$ is assigned a value which altogether will sum up to one.

Next the conditional distribution of a particular motion category $m_{i}$ given the part observed being $p_{j}$ is computed. With previously obtained similarity maps, the conditional probability can be represented as: 


$$
P_{k}\left(m_{i} \mid p_{j}\right)=\frac{\operatorname{SiMap}_{m_{i}, k}\left(p_{j}\right)}{\sum_{i=0}^{M} \operatorname{SiMap}_{m_{i}, k}\left(p_{j}\right)} .
$$

The conditional distribution of "motion" given "part" characterizes how likely the observed body part is performing a particular motion, assuming that the part is already identified as a given part.

Combining the joint distribution of part and motion, and the conditional distribution of motion given the part, the distribution of a part $p_{j}$ can be estimated using (17):

$$
P_{k}\left(p_{j}\right)=\frac{P_{k}\left(m_{i}, p_{j}\right)}{P_{k}\left(m_{i} \mid p_{j}\right)}=\frac{\sum_{i=0}^{M} \operatorname{SiMap}_{m_{i}, k}\left(p_{j}\right)}{\sum_{i=0}^{M} \sum_{j=0}^{W \times H} \operatorname{SiMap}_{m_{i}, k}\left(p_{j}\right)},
$$

in which $P_{k}\left(m_{i}, p_{j}\right)$ and $P_{k}\left(m_{i} \mid p_{j}\right)$ are calculated from (15) and (16), respectively. In Figure 4 the process from calculating the similarity map to part-distribution estimation for a particular $k$ (similarity measurement) is illustrated. On the left it shows multiple similarity maps $\operatorname{SiMap}_{m_{i}}\left(p_{j}\right)$ for different $m_{i}$, and on the right is the estimated part distribution $P\left(p_{j}\right)$. The

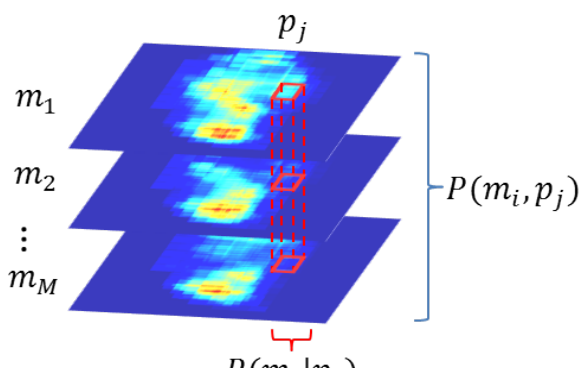

$P\left(m_{i} \mid p_{j}\right)$

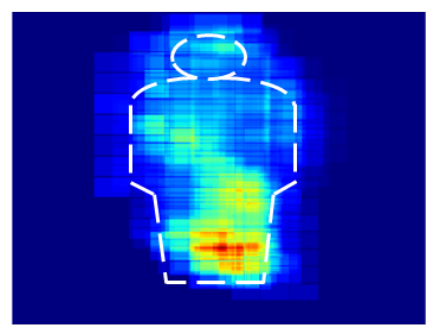

$P\left(p_{j}\right)$

Figure 4: From similarity maps to part distribution.

final part-distribution estimation is obtained by using the point-wise mean across all $k$. The fast part-distribution estimation gives a rough idea of which parts have high similarity and are thus less likely to violate the constraints.

When searching for the most violated constraints, the frame is split into grids of sub-frames, and a fixed number of pixel positions are sampled. The number of samples given to each subframe is based on the estimated part-distribution. A higher probability indicates a less likelihood 
of violation of the constraints, and thus less samples are given to the sub-frame. The most violated constraints are found using (13) in these samples.

\subsubsection{Inferencing Body Part and Motion}

Given a testing video $\left\{V_{I}\right\}$ with partial body observed and unknown motion, the goal is to determine which part it is represented by the bounding box in the canonical coordinates, and what motion the whole body is performing. The video is first represented by feature vector $x=F\left[V_{I}\right]$. Then it is plugged into the trained classifier to find its predicted label $y^{*}$ according to the following:

$$
y^{*}=\underset{y}{\arg \max }\langle w, \Psi(x, y)\rangle .
$$

The testing videos should also be evaluated against the reference videos at all positions from all motions categories, and the part and motion pair that maximizes the inner product of $w$ and $\Psi$ will be the inference result. Different from the training process, there are no multiple iterations like finding the most-violated constraints; therefore, a one-time exhaust search is all needed and feasible. In practice, however, we can accelerate the process by fine-grained uniform sampling with little compromise of accuracy.

\section{EXPERIMENTAL RESULTS}

\subsection{Experiment Setup}

The proposed approach is evaluated using both videos captured on a real robot as well as the UCF50 public dataset. Comprehensive experiments are conducted to evaluate the aspects of part identification and motion identification separately. Additionally, an experiment on applying our algorithm for the purpose of pose estimation is presented using the FLIC-motion dataset.

\subsubsection{WR Dataset Collected by Robot}

The robot platform used in our experiment is an intelligent wheelchair robot developed by SJTU [42] as shown in Figure 5a. The robot is equipped with a AR2407(TI) micro-processor and is powered by two $24 \mathrm{~V}$ batteries, weighted $60 \mathrm{~kg}$. The original physical size of the robot is 


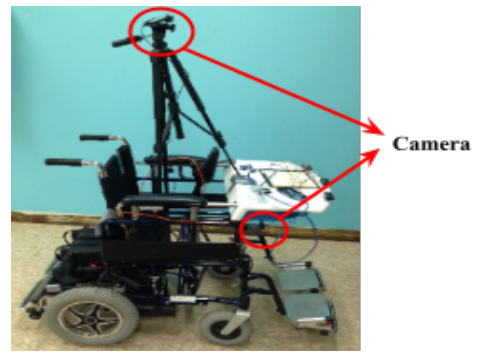

(a) Intelligent wheelchair robot with camera installed.

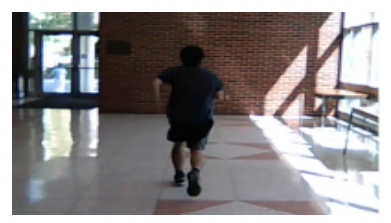

(b) Training video: full body running.

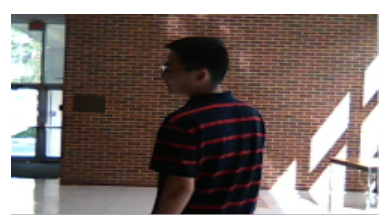

(c) Testing video: upper body turning-left.

Figure 5: Experiment setups.

$1160 \mathrm{~mm} \times 610 \mathrm{~mm} \times 950 \mathrm{~mm}$. A camera is mounted on the robot at a vertical height at 1.55 meters for video recording. During the experiments, the robot is controlled to follow a person at a $0.5-2.5$ meters distance.

Four different motion statuses are included: walking, running, turning-left and turning-right, which are commonly seen by human-following robots and important for robots to determine the moving tendency of the followed targets.

We collect two groups of videos that contain the fully observed body and one group of videos that contain the partially observed body to serve as reference, training and testing data, respectively. Snapshots of the collected video data are shown in Figure $5 \mathrm{~b}$ and $5 \mathrm{c}$. The data was collected from 5 individuals that are assumed to be the followed targets of the robot. Every person performs each of the motions for multiple times. In total there are 36 reference videos ( 9 for each motion), 12 videos for training, and 12 videos for testing. All the videos are recorded at $30 \mathrm{fps}$ frame rate and $320 \times 240$ resolution. This dataset collected by the wheelchair robot is referred to as the WR dataset in the following description.

\subsubsection{UCF50 Dataset}

We also test our approach in the UCF50 dataset [43], which is an action recognition dataset consisting of videos taken from YouTube. To simulate the video being recorded by human- 

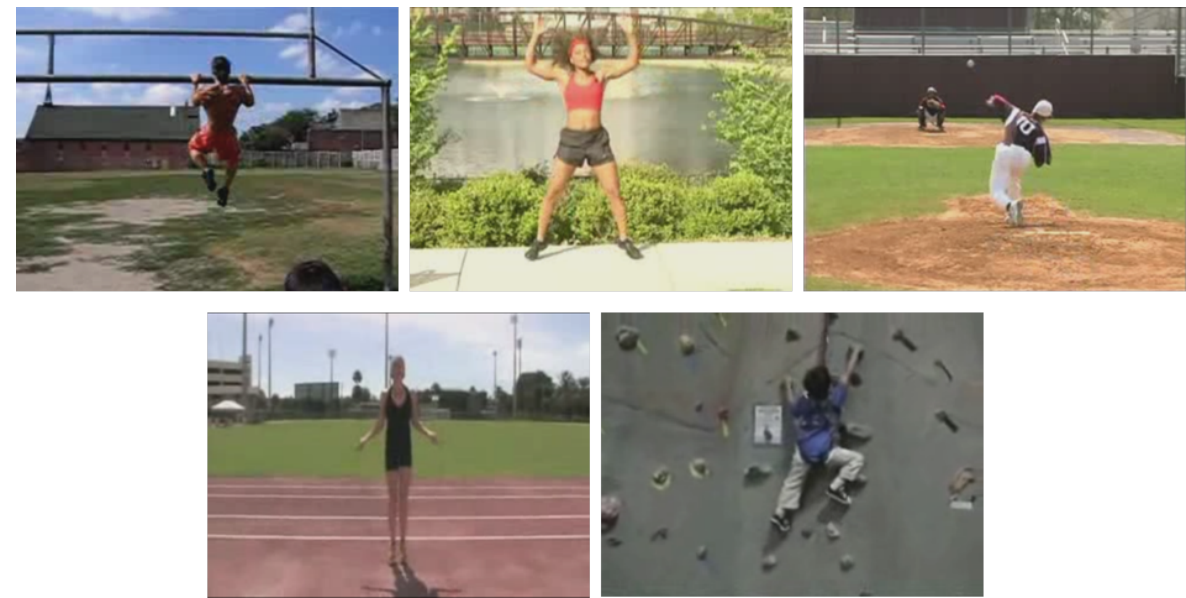

Figure 6: Examples of the UCF50 Dataset. First row from left to right: pulling-up, jumping-jack, baseball-pitch; second row from left to right: jumping-rope, rock-climbing.

following robots, the video clips should be recorded in a back or frontal (which is similar to back) view, and the videos should have corresponding full body videos available which serve as reference data. A subset of the whole database is selected based on the above criterion, including 5 motions: pulling-up, jumping-jack, baseball-pitch, jumping-rope and rock-climbing. Some of the examples from these datasets are shown in Figure 6. The length of the video clips ranges from 2 seconds to more than 20 seconds, with a resolution of $320 \times 240$. Altogether there are 119 videos, 45 of them are used as reference videos ( 9 of each motion), 35 as training and 39 as testing.
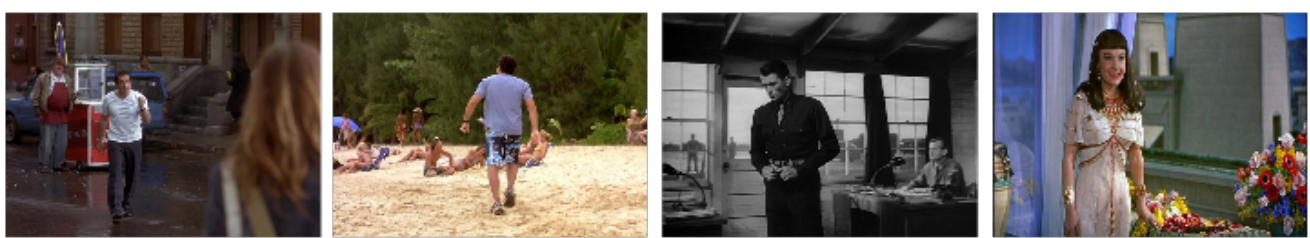

Figure 7: Examples of the FLIC-motion dataset.

\subsubsection{FLIC-motion Dataset}

The FLIC-motion dataset [15] is a set of 30 Hollywood movies from which the original FLIC dataset [14] of 5003 labeled RGB images were collected, to facilitate the usage of motion 
features for the task of pose-estimtion, as shown in Figure 7. The ground truth position of elbow and wrist are provided. A comparative experiment on modifying our algorithm for the purpose of pose estimation without considering motion identification is presented using this dataset.

\subsubsection{Parameters}

The parameters used in our experiments are stated here. For the sampled volumes, the size is $80 \times 60 \times 60$. The spatial-temporal sampling strides in estimating the part-distribution are $\Delta w=40, \Delta h=30$ and $\Delta l=30$. For the dense trajectory features, the same parameters used in [37] are applied. We cluster 200 visual vocabularies for BOW for each of the three low-level features. The clustering is performed multiple times and the group of vocabularies that produces the best result are kept. We sample 4000 times at each iteration when finding the most-violated constraints, and the size of each sub-frame is $20 \times 20$.

Note that the numbers of the training and testing videos are not equal to the number of training and testing samples. In the training process, the training samples are generated by sampling the training videos into spatial-temporal cubes with size $80 \times 60 \times 60$ and strides of 8,6 and 30 in column, row and temporal directions, respectively. The testing samples are generated in a similar way but with a larger stride of 12 and 9 in column and row directions, respectively. In our implementation, there are $J=9$ reference videos for each motion, $K=3$ different distance measurements and $N=3$ different low-level features. So each feature function in (9) is an 81dimensional vector. For the pose estimation in the FLIC-motion dataset, the number of reference videos is 12 , resulting in 108 -dimensional feature vectors.

\subsection{Results Analysis}

\subsubsection{Fast Part-distribution Estimation Guided Most Violated Constraints Searching}

Before examining the overall approach, we give an intuitive illustration of the intermediate results: the fast part-distribution estimation, which is used for reducing searching space for finding the most violated constraints in the optimization process. The three typical cases showing the upper body, lower body, and right side body are demonstrated in Figure 8. The left column shows the snapshots of the testing videos captured by the robot, and the right column shows the 

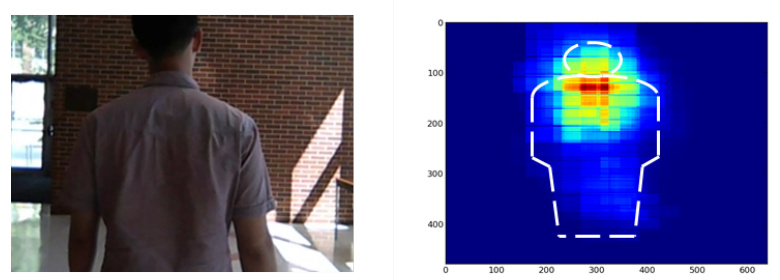

(a) Upper body case.
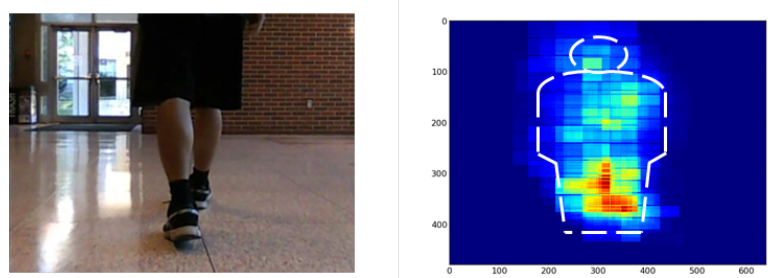

(b) Lower body case.
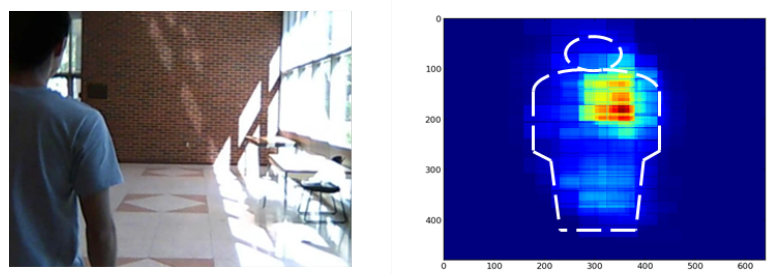

(c) Side body case.

Figure 8: Three typical cases and their part distributions with canonical virtual full human body overlaid: (a) Upper body, (b) Lower body, (c) Side body (best viewed in color).

visualized estimated body part distributions, with the canonical virtual full human body outline overlaid (white dashed silhouette).

From Figure 8 we can see that the probability is higher in the positions where the body parts are in the scene. These parts that are nearer to the correct position will be receiving less samples when searching for the most violated constraints.

\subsubsection{Part Identification}

In this section the performance of part identification is evaluated. The accuracy is reflected using both Bounding-box Overlap Rate (BOR), which is in part of the loss function as defined in (10) and the Mean Center Offset (MCO) describing the offset of centers of bounding boxes measured in pixels. The bounding boxes used to compute BOR and MCO are all aligned in the 
canonical coordinates, and pixels outside the canonical bounding box of the human body are not considered.

\begin{tabular}{|c|c|c|c|c||c|}
\hline & Walk & Run & $\begin{array}{c}\text { Turn- } \\
\text { Left }\end{array}$ & $\begin{array}{c}\text { Turn- } \\
\text { Right }\end{array}$ & All Motions \\
\hline BOR & $81.23 \%$ & $72.81 \%$ & $42.67 \%$ & $50.32 \%$ & $61.87 \%$ \\
\hline MCO & 12.95 & 17.68 & 40.83 & 33.50 & 26.12 \\
\hline
\end{tabular}

Table 1: Part accuracy of part identification in the WR dataset.

\begin{tabular}{|c|c|c|c|c|c||c|}
\hline & $\begin{array}{c}\text { Pull- } \\
\text { Up }\end{array}$ & $\begin{array}{c}\text { Jump- } \\
\text { Jack }\end{array}$ & $\begin{array}{c}\text { Baseball- } \\
\text { Pitch }\end{array}$ & $\begin{array}{c}\text { Jump- } \\
\text { Rope }\end{array}$ & $\begin{array}{c}\text { Rock- } \\
\text { Climb }\end{array}$ & All Motions \\
\hline BOR & $30.99 \%$ & $80.41 \%$ & $71.88 \%$ & $63.57 \%$ & $35.52 \%$ & $57.67 \%$ \\
\hline MCO & 58.58 & 12.98 & 19.74 & 27.55 & 49.79 & 32.65 \\
\hline
\end{tabular}

Table 2: Part accuracy of part identification in the UCF50 dataset.

From the tables we can see that the overall overlap rate of the bounding box is $61.87 \%$ in the WR dataset and $57.67 \%$ in the UCF50 dataset, and the offset of the center is in average 26.12 and 32.65 pixels in the two dataset, respectively. This should be able to provide a good estimation of what part is being observed. It can also be seen that the value differs among different motions significantly. For those motions that body positions are relatively still when performing, like Walk, the performance of part identification is significantly better than those requiring large body movements, for example PullUp. The reason is that the alignment can not be done accurately for the motions containing large body movements, which causes inaccurate labeling in the training process. Decreasing the alignment anchor interval can help improve the performance in such situations. Also, the movements in RockClimb do not have a routine pattern and are more random compared to motions with obvious patterns like JumpJack. This huge within-class deviation in body part positions causes difficulties in matching the observed part to correct position, resulting in large prediction error. A larger number of training samples that cover the different movements within a motion category more comprehensively may alleviate such problem. 


\subsubsection{Used as Pose Estimation}

A closely related but different problem is pose estimation. Among the simultaneous identification of both part and motion, the part aspect focus of our approach is to determine which part of the body is being observed, while the pose estimation aims at locating one or more specific body parts/joints among a larger picture that contains these body parts. In other words, our approach is to solve for "what", without any predefined model of a specific body part; while pose estimation is to solve for "where", usually with trained models of the particular body parts.

Our approach is not geared towards pose estimation. However, it could be interesting and insightful to see how it performs if used as a pose estimation method. Since we describe the body parts using relative positions with respect to the whole body in canonical coordinates, this can be achieved with some modifications on the decision making part. A comparative experiment is conducted following the set up in [15] using the FLIC-motion dataset. For comparision purpose, the same process of applying empirically calculated joint-masks which describes the possible joint locations and the rough torso location is used to constrain the searching space in optimization. For each testing video, it is densely sampled into spatial-temporal cubes. Both the predicted labels of each cube and the ground truth of elbow and wrist joints are mapped onto the canonical coordinates. The mean position of elbow and wrist are learned from the training videos. The cube whose predicted label is nearest to the mean positions of elbow/wrist is selected as the decision of elbow/wrist, and corresponding error is measured using the distance between the ground truth of elbow/wrist, and the ground truth label of the selected cube. The results are shown in Figure 9. The state-of-the-art pose estimation algorithm MoDeep [15] is used for comparison, together with MODEC [14], and the approach by Eichner et al. [13]. It is surprising to discover that our approach performs comparable to MoDeep and better than the other algorithms in the higher error radius threshold region, but is outperformed by MoDeep in the high precision region. Recall that our approach is not designed for pose estimation and is towards general part identification instead of localizing some particular parts like elbow and wrist, thus it is not specifically trained for these parts; our algorithm is more of a patch matching than point matching, thus when error radius increases, our performance increases rapidly. For all the 


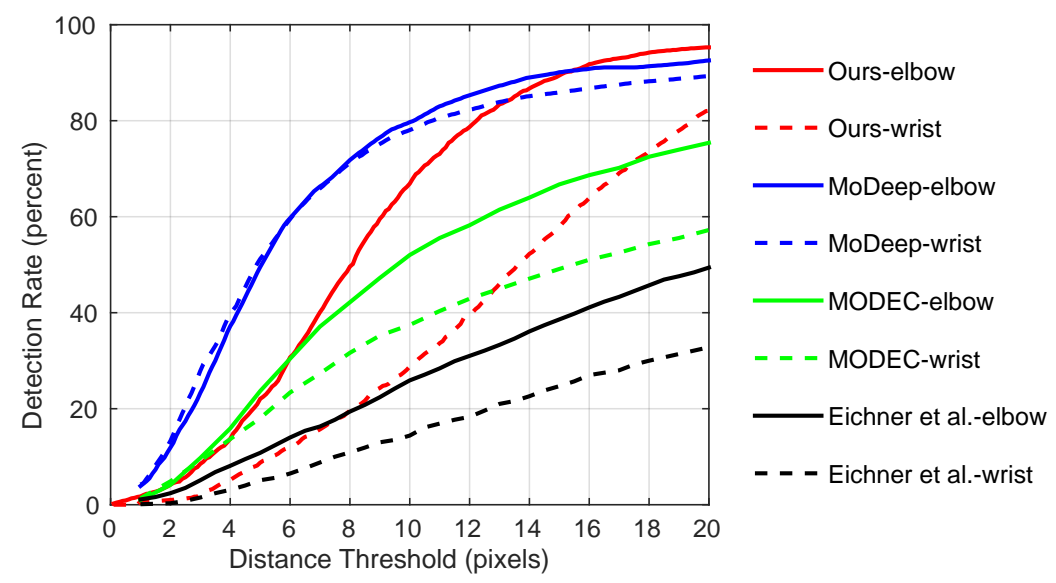

Figure 9: Comparative results of our approach used as pose estimation.

methods, the detection rate for elbow is higher than that of wrist, due to the greater freedom of movement for the wrist.

\subsubsection{Motion Identification}

We also evaluate the accuracy of motion identification achieved under limited perception of the human body. As a comparison, a multiclass SVM (MCSVM) are trained using the same low-level features (IDT features in [37]), however, with only motion labels instead of both part and motion labels. Since both methods share the same low-level features, the main focus is on the improvement of our approach for the cases of limited perception of human body. The comparative results of motion identification accuracies are shown in Figure 11. We further present the confusion matrices of our approach in both datasets in Figure 10, to investigate the detailed performance for different motions.

In the confusion matrix for WR dataset, Run has the best performance. One possible reason is that Run has the most unique motion pace that is faster than the other three motions, and such uniqueness in motion spreads across almost all the body parts. In contrast, PullUp and RockClimb in UCF50 dataset are confused with JumpJack and BaseballPitch to some degree, 


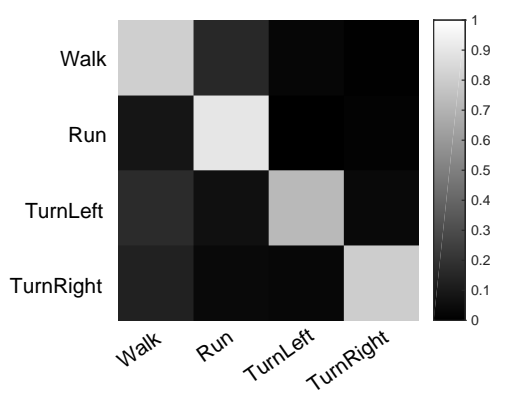

(a) Confusion matrix for WR dataset.

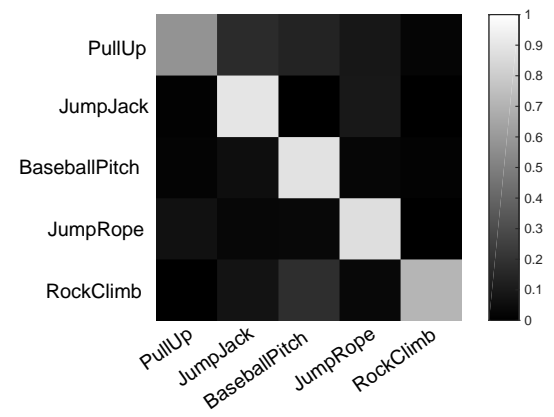

(b) Confusion matrix for UCF50 dataset.

Figure 10: Confusion matrices for 2 databases.

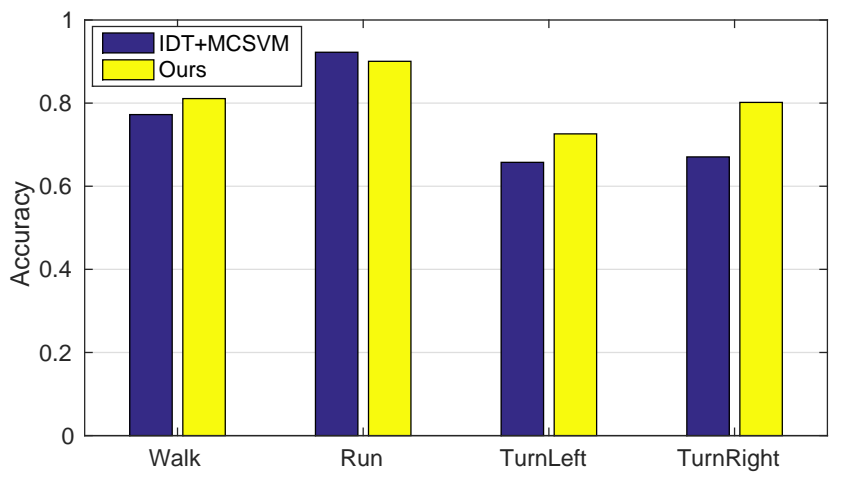

(a) Motion accuracy for WR dataset.

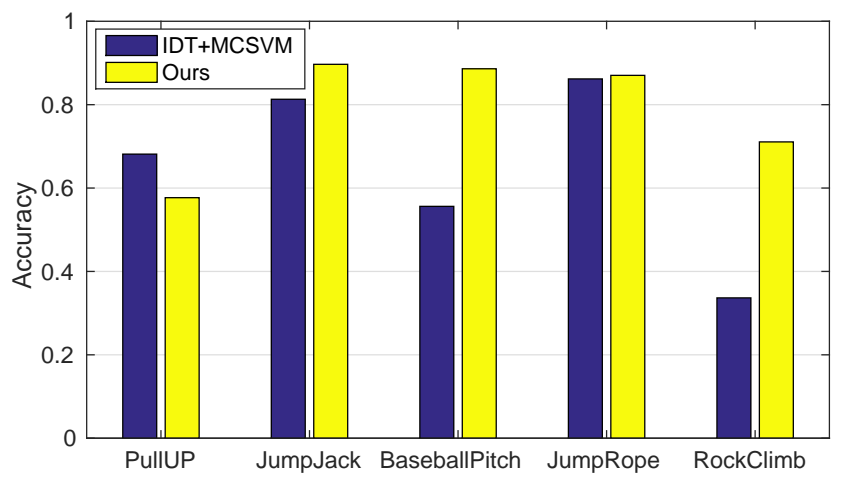

(b) Motion accuracy for UCF50 dataset.

Figure 11: Accuracy of motion identification in 2 databases. 
respectively. Some body part like torso and head may look similar in both PullUp and JumpJack, while the similarity from RockClimb and BaseballPitch is more likely to come from the semistatic moments during the motion.

The overall accuracy of motion identification is $81.16 \%$ and $75.65 \%$ for our approach and the comparative method in the WR dataset, and the numbers are $79.93 \%$ and $64.53 \%$ in the UCF50 dataset. From Figure 11 we can see that our approach performs better with partial body observed, which implies that considering part and motion simultaneously helps reduce the ambiguity. We also notice that for the motion PullUp in the UCF50 dataset, our approach has worse performance. Comparing this to its part identification performance, we can see that failure in identifying the body parts affects the ability to identify the motion.

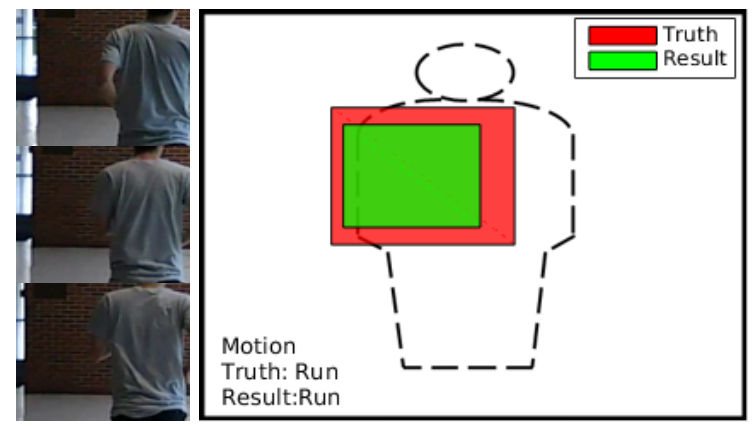

(a) Upper left body in running motion.

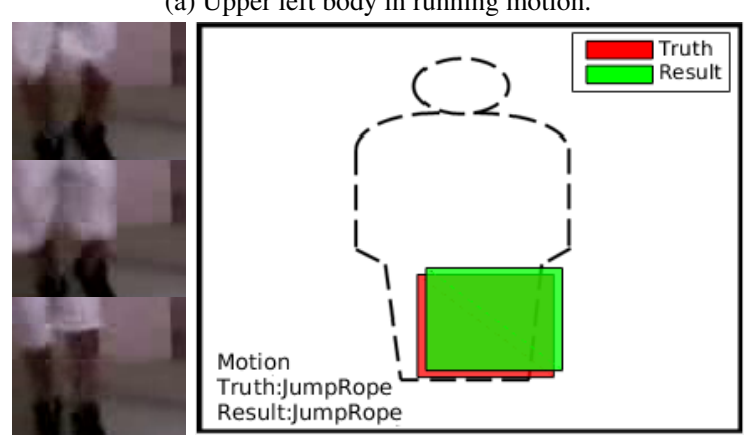

(b) Lower right body doing jumping rope.

Figure 12: Left column: snapshots from the observed videos; right column: the visualized results.

The overall output of our approach is the combination of body part and motion, as some examples of visualized results are shown in Figure 12. The left column shows the observed body part and 3 frames are presented illustrating the undergoing motion, while the right column shows 
the ground truth part in red rectangle and our predicted part in green rectangle.

\subsubsection{Computational Cost}

We implement our approach in $\mathrm{C}++$ and test the computational time using a $3.0 \mathrm{GHz} \mathrm{CPU}$ with 8 cores, and the operating system is Ubuntu 12.04. For each cube which is treated as one testing partially observed video, it takes 2.4 seconds to predict the label on average. The lowlevel feature extraction part consumes 0.8 second and the label infering process takes 1.6 seconds. This is compared to approximately 1 second for IDT+MCSVM (for motion identification) and MODEC [14], 2 seconds for MoDeep [15], and 8 seconds for Eichner et al. [13], as reported. Note that these only serve as a reference because different implementations and hardware platforms affect the resulting computational time. The parallelization and ultilization of GPU, for example, will further decrease the time consumption, as implemented in MoDeep.

\section{CONCLUSIONS}

We have presented an approach for simultaneous identification of human body part and motion for human-following robots. We formulate the problem in a structured learning framework, by regarding part and motion as labels in the structured output space and introducing a reference set. Detailed design of the structured SVM and its implementation are presented.

The proposed approach are able to achieve the following simultaneously: 1. Identifying a random portion of the body that appears in the video and no part-specific model for each separate part is needed; 2 . Identifying the whole body motion being performed from partially observed body. The approach is evaluated using real videos captured by a wheelchair robot following different subjects as well as public datasets. The results verify the effectiveness of the new approach.

\section{ACKNOWLEDGMENTS}

This work is supported by the National Science Foundation under grant CNS-1218876. 


\section{References}

[1] A. Cosgun, D. A. Florencio, H. I. Christensen, Autonomous person following for telepresence robots, in: Proc. IEEE International Conference on Robotics and Automation (ICRA), 2013, pp. 4335-4342.

[2] T. Horiuchi, S. Thompson, S. Kagami, Y. Ehara, Pedestrian tracking from a mobile robot using a laser range finder, in: Proc. IEEE International Conference on Systems, Man and Cybernetics (ISIC), 2007, pp. 931-936.

[3] M. Kobilarov, G. Sukhatme, J. Hyams, P. Batavia, People tracking and following with mobile robot using an omnidirectional camera and a laser, in: Proc. IEEE International Conference on Robotics and Automation (ICRA), 2006, pp. 557-562.

[4] J. Satake, J. Miura, Robust stereo-based person detection and tracking for a person following robot, in: Proc. IEEE International Conference on Robotics and Automation Workshop (ICRAW), 2009.

[5] R. Gockley, J. Forlizzi, R. Simmons, Natural person-following behavior for social robots, in: Proc. International Conference on Human-robot interaction, ACM, 2007, pp. 17-24.

[6] Boston Dynamics, Ls3 - legged squad support systems, http://www.bostondynamics.com/robot_ls3.html.

[7] J. Brookshire, Person following using histograms of oriented gradients, International journal of social robotics 2 (2) (2010) 137-146.

[8] I. Ardiyanto, J. Miura, Partial least squares-based human upper body orientation estimation with combined detection and tracking, Image and Vision Computing 32 (2014) 904-915.

[9] B. Wu, R. Nevatia, Detection and tracking of multiple, partially occluded humans by bayesian combination of edgelet based part detectors, International Journal of Computer Vision 75 (2) (2007) 247-266.

[10] P. Srinivasan, J. Shi, Bottom-up recognition and parsing of the human body, in: Energy Minimization Methods in Computer Vision and Pattern Recognition, Springer, 2007, pp. 153-168.

[11] C. Plagemann, V. Ganapathi, D. Koller, S. Thrun, Real-time identification and localization of body parts from depth images, in: Proc. IEEE International Conference on Robotics and Automation (ICRA), 2010, pp. 3108-3113.

[12] P. F. Felzenszwalb, R. B. Girshick, D. McAllester, D. Ramanan, Object detection with discriminatively trained part-based models, Pattern Analysis and Machine Intelligence, IEEE Transactions on 32 (9) (2010) 1627-1645.

[13] M. Eichner, V. Ferrari, S. Zurich, Better appearance models for pictorial structures, in: Proc. The British Machine Vision Conference (BMVC), Vol. 2, 2009.

[14] B. Sapp, B. Taskar, Modec: Multimodal decomposable models for human pose estimation, in: Proc. IEEE Conference on Computer Vision and Pattern Recognition (CVPR), 2013, pp. 3674-3681.

[15] A. Jain, J. Tompson, Y. LeCun, C. Bregler, Modeep: A deep learning framework using motion features for human pose estimation, in: Proc. The Asian Conference on Computer Vision (ACCV), Springer, 2014, pp. 302-315.

[16] I. Laptev, On space-time interest points, International Journal of Computer Vision 64 (2-3) (2005) 107-123.

[17] P. Dollár, V. Rabaud, G. Cottrell, S. Belongie, Behavior recognition via sparse spatio-temporal features, in: Visual Surveillance and Performance Evaluation of Tracking and Surveillance, Joint IEEE International Workshop on, 2005 , pp. 65-72. 
[18] H. Wang, A. Klaser, C. Schmid, C.-L. Liu, Action recognition by dense trajectories, in: Proc. IEEE Conference on Computer Vision and Pattern Recognition (CVPR), 2011, pp. 3169-3176.

[19] Y. Zhu, N. M. Nayak, A. K. Roy-Chowdhury, Context-aware modeling and recognition of activities in video, in: Proc. IEEE Conference on Computer Vision and Pattern Recognition (CVPR), 2013, pp. 2491-2498.

[20] D. Oneata, J. Verbeek, C. Schmid, Action and event recognition with fisher vectors on a compact feature set, in: Proc. IEEE Conference on International Conferene on Computer Vision (ICCV), 2013, pp. 1817-1824.

[21] H. Pirsiavash, D. Ramanan, Parsing videos of actions with segmental grammars, in: Proc. IEEE Conference on Computer Vision and Pattern Recognition (CVPR), 2014, pp. 612-619.

[22] W. Gong, J. Gonzàlez, J. M. R. Tavares, F. X. Roca, A new image dataset on human interactions, in: Articulated Motion and Deformable Objects, Vol. 7378, pp. 204-209.

[23] Y. Cao, D. Barrett, A. Barbu, S. Narayanaswamy, H. Yu, A. Michaux, Y. Lin, S. Dickinson, J. M. Siskind, S. Wang, Recognize human activities from partially observed videos, in: Proc. IEEE Conference on Computer Vision and Pattern Recognition (CVPR), 2013, pp. 2658-2665.

[24] S. Bambach, A survey on recent advances of computer vision algorithms for egocentric video, in: Computing Research Repository of arxiv, 2015, http://arxiv.org/abs/1501.02825.

[25] X. Ren, C. Gu, Figure-ground segmentation improves handled object recognition in egocentric video, in: Proc. IEEE Conference on Computer Vision and Pattern Recognition (CVPR), 2010, pp. 3137-3144.

[26] A. Fathi, X. Ren, J. M. Rehg, Learning to recognize objects in egocentric activities, in: Proc. IEEE Conference On Computer Vision and Pattern Recognition (CVPR), 2011, pp. 3281-3288.

[27] H. Pirsiavash, D. Ramanan, Detecting activities of daily living in first-person camera views, in: Proc. IEEE Conference on Computer Vision and Pattern Recognition (CVPR), 2012, pp. 2847-2854.

[28] M. S. Ryoo, L. Matthies, First-person activity recognition: What are they doing to me?, in: Proc. IEEE Conference on Computer Vision and Pattern Recognition (CVPR), 2013, pp. 2730-2737.

[29] Z. Lu, K. Grauman, Story-driven summarization for egocentric video, in: Proc. IEEE Conference on Computer Vision and Pattern Recognition (CVPR), 2013, pp. 2714-2721.

[30] Y. Poleg, C. Arora, S. Peleg, Temporal segmentation of egocentric videos, in: Proc. IEEE Conference on Computer Vision and Pattern Recognition (CVPR), 2014, pp. 2537-2544.

[31] A. Betancourt, M. M. López, C. S. Regazzoni, M. Rauterberg, A sequential classifier for hand detection in the framework of egocentric vision, in: Proc. IEEE Conference on Computer Vision and Pattern Recognition Workshops (CVPRW), 2014, pp. 600-605.

[32] S. Lee, S. Bambach, D. J. Crandall, J. M. Franchak, C. Yu, This hand is my hand: A probabilistic approach to hand disambiguation in egocentric video, in: Proc. IEEE Conference on Computer Vision and Pattern Recognition Workshops (CVPRW), IEEE, 2014, pp. 557-564.

[33] I. Tsochantaridis, T. Hofmann, T. Joachims, Y. Altun, Support vector machine learning for interdependent and structured output spaces, in: Proc. of the twenty-first international conference on Machine learning, ACM, 2004, 
p. 104.

[34] M. B. Blaschko, C. H. Lampert, Learning to localize objects with structured output regression, in: Proc. European Conference on Computer Vision (ECCV), Springer, 2008, pp. 2-15.

[35] N. Hu, Z. Lou, G. Englebienne, B. Kröse, Learning to recognize human activities from soft labeled data, Robotics: Science and Systems (RSS). IEEE.

[36] L. Bertelli, T. Yu, D. Vu, B. Gokturk, Kernelized structural svm learning for supervised object segmentation, in: Proc. IEEE Conference on Computer Vision and Pattern Recognition (CVPR), 2011, pp. 2153-2160.

[37] H. Wang, C. Schmid, Action recognition with improved trajectories, in: Proc. IEEE International Conference on Computer Vision (ICCV), 2013, pp. 3551-3558.

[38] N. Dalal, B. Triggs, C. Schmid, Human detection using oriented histograms of flow and appearance, in: Proc. European Conference on Computer Vision (ECCV), Springer, 2006, pp. 428-441.

[39] R. Szeliski, Image alignment and stitching: A tutorial, Foundations and Trends in Computer Graphics and Vision 2 (2006) 1-104.

[40] M. A. Fischler, R. C. Bolles, Random sample consensus: a paradigm for model fitting with applications to image analysis and automated cartography, Communications of the ACM 24 (6) (1981) 381-395.

[41] L. Fei-Fei, R. Fergus, A. Torralba, Recognizing and learning object categories, CVPR Short Course.

[42] Intelligent wheelchair robot, http://robotics.sjtu.edu.cn.

[43] K. K. Reddy, M. Shah, Recognizing 50 human action categories of web videos, Machine Vision and Applications 24 (5) (2013) 971-981. 